\title{
CLINICAL RESEARCH ARTICLE The effect of unilateral stroke on autonomic function in the term newborn
}

\author{
Daniel A. Reich ${ }^{1}$, Rathinaswamy B. Govindan ${ }^{1}$, Matthew T. Whitehead ${ }^{2}$, Jichuan Wang ${ }^{3}$, Taeun Chang $^{4}$, Srinivas Kota ${ }^{1}$ and \\ Adre J. du Plessis ${ }^{1}$
}

BACKGROUND: The mature cerebral cortex has a topographically organized influence on reflex autonomic centers of the brainstem and diencephalon and sympathetic activation coming primarily from the right hemisphere and parasympathetic activation from the left. In the term newborn, the maturational status of this central autonomic system remains poorly understood. METHODS: Sixteen term newborns admitted to Children's National with unilateral middle cerebral artery (MCA) strokes $(n=8$ left, $n=8$ right) had archived continuous electrocardiograph (EKG) signals available. We compared stroke laterality and severity with indices of autonomic function, as measured by heart rate variability. We performed both time- and frequency-domain analyses on the R-R interval (RRi) over 24h of continuous EKG data at around 7 days of age.

RESULTS: Right MCA stroke significantly increased sympathetic tone, while left MCA stroke increased parasympathetic tone. Regardless of laterality, stroke severity was associated inversely with sympathetic tone and positively with parasympathetic tone. Surprisingly, injury to either insular region had no significant autonomic effect. Phenobarbital blood levels were positively associated with sympathetic tone and inversely related to parasympathetic tone.

CONCLUSION: Based on these findings, it is difficult to reconcile the functional topography of the central autonomic system in term newborns with that currently proposed for the normal mature brain. Further investigation is clearly needed.

Pediatric Research (2019) 85:830-834; https://doi.org/10.1038/s41390-019-0320-0

\section{INTRODUCTION}

Hypoxic-ischemic insults play a prominent role in perinatal brain injury. ${ }^{1}$ The cerebral hemispheres are more vulnerable to such injury than the brainstem. ${ }^{1}$ Reflexive autonomic centers that modulate heart rate and its variability are located in the brainstem and diencephalon and are functional before normal term gestation. $^{2}$ During maturation these brainstem centers are recruited into a complex network of supratentorial centers that modulate their reflexive activity. ${ }^{3}$ The maturational timeline for this central autonomic network is not well understood.

Currently, fetal heart rate remains the only readily available continuous physiological signal for clinical intrapartum and prenatal fetal monitoring. However, whether management based on this technique reduces intrapartum brain injury remains controversial, for a variety of reasons. ${ }^{4}$ One such reason is an incomplete understanding of the maturational status at term gestation of the autonomic centers in the more vulnerable cerebral hemispheres, and their relationship with autonomic centers in the more robust brainstem and diencephalon. New insights into the relationship between cerebral cortical and brainstem autonomic centers might enhance the diagnostic utility of fetal heart rate monitoring for detecting imminent insult to the immature brain. A central goal of this study, and others in our program, is to characterize the relationship between the higher cerebral regions and brainstem centers of autonomic control in the term newborn.
Neonatal stroke is primarily vaso-occlusive and most commonly involves the middle cerebral artery (MCA) territories, left more than right. ${ }^{1}$ For these reasons, we used a unilateral neonatal MCA stroke model, in which the opposite hemisphere is apparently intact, to determine the effect of stroke laterality and severity on autonomic function. Our central hypothesis is that the lateralization of the MCA infarct will have differential effect on autonomic tone, based on metrics of heart rate variability (HRV).

\section{METHODS}

The Children's National Neonatal Intensive Care Unit (NICU) is a major regional (Level IV) referral center for critically ill newborn infants, including those with stroke and seizures. The Children's National Medical Center Institutional Review Board approved the retrospective analysis of the data. We identified 16 term newborns with unilateral MCA strokes on either the left $(n=8)$ or right side $(n=8)$ for whom we had archived continuous electrocardiograph (EKG) data from their bedside monitor during their NICU admission. MCA stroke/infarction from detailed characterization of magnetic resonance imaging (MRI) was defined qualitatively as confluent signal abnormality and reduced diffusion involving either the entire MCA vascular territory or a segment of it, as determined by an experienced board-certified pediatric neuroradiologist. The clinical data, including gender, gestational age at

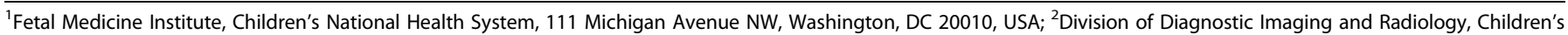

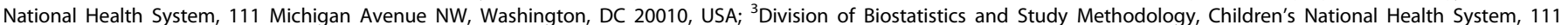
Michigan Avenue NW, Washington, DC 20010, USA and ${ }^{4}$ Division of Neurology, Children's National Health System, 111 Michigan Avenue NW, Washington, DC 20010, USA Correspondence: Adre J. du Plessis (adupless@childrensnational.org) 
birth, and seizure medications, were extracted from a clinical database. We selected the MCA because it is by far the most common territory for neonatal stroke, and in the mature brain it is known to contain important cortical autonomic centers, including the insula. ${ }^{5}$ The severity of MCA stroke was classified according to the previously validated Modified Pediatric ASPECT (Alberta stroke program early CT) score ${ }^{6}$ based on the ability of this instrument to quantify childhood arterial ischemic stroke volumes. Higher scores (maximum of 15 points per hemisphere) indicate larger areas of infarction. ${ }^{6}$ In this study, there was a strong positive correlation between the Modified Pediatric ASPECT scores and infarct volumes in neonates with excellent interrater reliability among three readers (a board-certified pediatric neuroradiologist, a pediatric neuroradiology fellow, and a pediatric neurologist).

All the newborns received prolonged continuous video electroencephalograms (cEEG) on admission as per routine clinical protocol to detect and treat seizures. EEG-confirmed seizures were treated with intravenous phenobarbital as the first-line antiseizure medication (ASM). Persistent EEG seizures were treated with fosphenytoin or levetiracetam. Status epilepticus was treated with midazolam infusions. All ASMs except for phenobarbital were discontinued prior to discharge. Midazolam infusions were discontinued within $48 \mathrm{~h}$ of achieving seizure cessation. As per American Clinical Neurophysiology Society guidelines, cEEG was performed until seizure-free for at least $24 \mathrm{~h}$.

Electrocardiographs (EKGs) offer a convenient means of studying the autonomic nervous system (ANS) at the bedside. Heart rate is the result of complex interactions between sympathetic and parasympathetic tone. The beat-to-beat interval calculated from the EKG provides a high temporal resolution by which to study the fluctuations in heart rate. Using both time and frequency domain ${ }^{7-9}$ approaches it is possible to characterize activity in the two arms of the ANS. We retrieved $24 \mathrm{~h}$ of EKG data sets from all of the infants on or around their seventh day of life. In all cases, newborns had been seizure-free for at least $48 \mathrm{~h}$ and ASMs discontinued, except for phenobarbital. None of the neonates received blood pressure medications during their NICU course. The sampling rate of EKG was $125 \mathrm{~Hz}$ and beat-to-beat interval (RRi) was calculated. For spectral analysis, the RRi was converted into uniformly sampled data using the cubic spline interpolation technique with a sample rate of $5 \mathrm{~Hz}$. We partitioned the RRi into 10-min epochs and quantified frequency and time domain HRV metrics. We used the Welch periodogram approach to estimate the power spectrum of RRi. In brief, the RRi was divided into 30-s epochs. For RRi in each epoch, a periodogram was calculated as the square of the magnitude of the Fourier transform of RRi. To this end, the power spectral estimate was obtained as the average of the periodograms from all epochs. Using the power spectrum, the low-frequency (LF) and highfrequency (HF) metrics were calculated as the median of the logarithm of spectral power in $0.05-0.25$ and $0.3-1 \mathrm{~Hz}$, respectively. The normalized LF (nLF) $(0.05-0.25 \mathrm{~Hz})$ and normalized HF (nHF) $(0.3-1 \mathrm{~Hz})$ were calculated as the sum of the spectral powers in their respective frequency bands, and they were normalized by total power, which is the sum of the spectral powers in the $0.05-2 \mathrm{~Hz}$ band. $^{10}$

Kubios HRV (Kubios; Kuopio, Finland) is an HRV analysis opensource software tool that allows "cleaning" of the EKG, calculation of the beat-to-beat interval (RRi), and characterization of HRV with power spectral analysis, detrended fluctuation analysis (DFA), and information theoretic-based approaches. These tools are developed in the MATLAB (Mathworks, Natick, MA) environment. We adapted the analysis tools to account for non-stationary data from ICU environment. ${ }^{11}$ Specifically, we modified the power spectral analysis $^{8}$ and DFA ${ }^{7}$ to mitigate the effect of non-stationarity on variability analysis. These modifications were developed in the MATLAB environment, and have been used in several of our published studies. ${ }^{10,12}$
The time-domain metrics were characterized using a DFA. ${ }^{7}$ DFA is a modified root mean square (RMS) analysis. In DFA, the mean value of RRi is subtracted and the following steps are taken: (1) a profile function is obtained as the cumulative sum of the RRi; (2) the profile is partitioned into epochs containing " $s$ " beats; (3) the profile inside each epoch is fitted using a fourth order polynomial and the RMS of the profile from the best fit is calculated as the local fluctuation function; (4) the median of the local fluctuation function is calculated to obtain global fluctuation function. Steps 2-4 are repeated for different " $s$ " values. Using the global fluctuation function, the following metrics were obtained: $a_{1}, a_{2}$, $R M S_{1}$, and RMS 2 . The $a_{1}$ and $a_{2}$ metrics were calculated for 15-30 and $35-150$ beats, respectively. The a metrics was obtained as the slope of the fluctuation function vs. "s" in double logarithmic representation, in the respective scaling regions. The RMS1 and RMS2 metrics were calculated for $15-50$ and $100-150$ beats, respectively. ${ }^{7}$ The RMS metrics were obtained as the maximum of the fluctuation function in the respective scaling regions. ${ }^{7}$ The a metrics characterize the autocorrelation in RRi, whereas the RMS metrics characterize the variability in RRi. The time domain metrics, as well as nLF and LF, characterize the sympathetic and baroreceptor-mediated parasympathetic tone of the ANS. The nHF and HF characterize the parasympathetic tone of the ANS. $a_{2}$ is a measure of variability in the ultralow frequency.

For every subject, each HRV metric was averaged over all $24 \mathrm{~h}$.

Statistical analysis

Descriptive statistics were used to characterize the sample with regards to the quantified HRV metrics (i.e., $a_{1}, a_{2}, R M S 1, R M S 2, n L F$, $\mathrm{nHF}, \mathrm{LF}, \mathrm{HF}$, and the ratio of LF over HF), as well as the stroke-related factors, such as the severity of the stroke, the location of the stroke in an insular region, and the use of phenobarbital. The manner in which the stroke-related factors would affect each of the quantified HRV metrics was assessed using multiple regression.

We evaluated the effects of stroke laterality and severity, as well as the effects of insular injury, and the use of ASM on each HRV metric using separate multiple regressions. Injury in the insular region was recoded as a dichotomous variable (any insular injury vs. none). All 16 neonates were initially treated with phenobarbital. For seizures refractory to phenobarbital, nine also received fosphenytoin, of which two also received levetiracetam and two also received midazolam infusion for status epilepticus on EEG. At the time of HRV analysis, all subjects were weaned to phenobarbital only. Given our sample size, we considered only phenobarbital levels in our models.

To examine whether and how stroke severity would moderate the effect of stroke laterality, we tested the interaction between laterality and severity in each model. To test this interaction, severity was recoded as the deviation from the grand mean level of severity of the overall sample. As such, the main effect of laterality can be interpreted as the effect of laterality, corresponding to the mean level of severity. When the interaction effect was statistically significant, the corresponding main effect, whether statistically significant or not, was kept in the model and included in the calculation of the effect of the corresponding variable. ${ }^{13}$ If the interaction was not statistically significant, it was removed from the model.

Finally, we determined whether stroke laterality influenced those hemodynamic indices under autonomic control by comparing averaged values for heart rate, systolic, diastolic, and mean arterial blood pressure for left vs. right MCA stroke over the same $24 \mathrm{~h}$ period from which the HRV indices were derived. Statistical significance was determined by simple $T$ tests.

All models were estimated using Mplus 8.0. ${ }^{14}$ Under the condition of data non-normality due to the small sample size, robust maximum likelihood estimator was used for model estimation, providing parameter estimates with standard errors that are robust to data non-normality. ${ }^{14}$ 


\begin{tabular}{|ll|}
\hline Table 1. Patient characteristics $(n=16)$ & \\
\hline Male, $n$ (\%) & $9(56.3 \%)$ \\
Gestational age (weeks), mean \pm s.d. & $39.0 \pm 1.2$ \\
Mode of delivery, $n$ (\%) & $10(62.5 \%)$ \\
$\quad$ Cesarean & $6(37.5 \%)$ \\
$\quad$ Vaginal & \\
Apgar scores, median (range) & $8(2-9)$ \\
$\quad 1$ min & $9(4-9)$ \\
$\quad 5$ min & $3.58 \pm 0.665$ \\
Birth weight (kg), mean \pm s.d. & $16(100 \%)$ \\
Seizures, $n$ (\%) & $11(69 \%)$ \\
$\quad$ Clinical & \\
$\quad$ EEG confirmed & $16(100 \%)$ \\
Medications during study time frame, $n$ (\%) & $9(56 \%)$ \\
$\quad$ Phenobarbital & $2(12.5 \%)$ \\
$\quad$ Fosphenytoin & $2(12.5 \%)$ \\
$\quad$ Levetiracetam & \\
$\quad$ Midazolam infusion & \\
\hline EEG electroencephalogram \\
aFive patients received phenobarbital for clinical seizures before going on \\
EEG recording
\end{tabular}

\section{RESULTS}

The clinical characteristics of the study population are shown in Table 1. A summary of HRV metrics is given in Table 2 . The grand mean stroke severity level by the ASPECT score (Table 3$)^{6}$ was 5.313 (2.12), recoded as zero for subsequent analyses. The mean severity was slightly higher in the left hemisphere than in the right hemisphere (4.5 vs. 6.125, $P=0.06)$. The mean serum level of phenobarbital on or around their seventh day of life was 38.9 (5.7).

\section{Statistical results}

Laterality had a significant positive effect on $a_{1}(0.227, P=0.028)$; that is, $a_{1}$ level is about 0.227 higher among patients with right MCA stroke, compared to those with left MCA stroke. The effect of stroke laterality on $a_{2}$ was not statistically significant $(0.036, P=$ 0.478 ), but stroke laterality has a significant positive interaction with stroke severity $(0.058, P=0.001)$, and as such, stroke laterality has a significant positive effect on $a_{2}(0.094, P=0.017)$. Stroke laterality had a marginally significant negative effect on $\mathrm{nHF}$ $(-0.091, P=0.069)$, but no significant effect on other HRV metrics.

Stroke severity had a negative effect on $a_{1}(-0.069, P=0.006)$, suggesting that stroke severity is associated with a reduction in the sympathetic tone. Conversely, stroke severity has a positive effect on nHF $(0.024, P=0.034)$, suggesting an increase in parasympathetic tone when stroke severity measure is one unit above its average level. The main effect of stroke severity on $a_{2}$ is negative, but not statistically significant $(-0.014, P=0.221)$; however, severity has a significant interaction with laterality $(0.058, P=0.001)$; thus, its effect on $a_{2}$ is positive and significant $(0.044, P=0.001)$. Stroke in the insular region of either side has no significant effect on any of the HRV metrics.

Phenobarbital blood level has a significant effect on a number of the HRV metrics, including (i) a positive effect on the sympathetic metrics, $a_{1}(0.01, P=0.032)$ and $n L F(0.008, P=$ $0.007)$, (ii) a negative impact on the parasympathetic metrics, nHF $(-0.008, P=0.003)$ and HF $(0.024, P=0.069)$, and (iii) a negative effect on the sympathovagal ratio of LF/HF $(-0.003, P=0.037)$. Together, these results suggest that phenobarbital increases sympathetic tone and reduces parasympathetic tone in the newborn with stroke.

\section{Table 2. HRV summary}

\begin{tabular}{lc}
\hline HRV metric & Mean \pm standard deviation \\
\hline$\alpha_{1}$ & $0.92 \pm 0.29$ \\
$\alpha_{2}$ & $1.13 \pm 0.13$ \\
RMS1 & $0.017 \pm 0.009$ \\
RMS2 & $0.062 \pm 0.025$ \\
$\mathrm{nLF}$ & $0.558 \pm 0.139$ \\
$\mathrm{nHF}$ & $0.374 \pm 0.113$ \\
$\mathrm{LF}$ & $-3.280 \pm 0.511$ \\
$\mathrm{HF}$ & $-3.930 \pm 0.410$ \\
$\mathrm{nLF} / \mathrm{nHF}$ & $1.706 \pm 0.804$ \\
\hline
\end{tabular}

$\alpha_{1}, \alpha_{2}, \mathrm{nLF}, \mathrm{nHF}$, and $\mathrm{nLF} / \mathrm{nHF}$ are dimensionless quantities. The unit of RMS1 and RMS2 is second. LF and HF are logarithm transformed spectral powers

HRV heart rate variability, $n L F$ normalized low frequency, $n H F$ normalized high frequency, RMS root mean square

Table 3. Stroke severity using ASPECT score ${ }^{6}$ in newborn infants with left $(n=8)$ and right $(n=8)$ MCA strokes

\begin{tabular}{lc}
\hline Age (days) at MRI, median (range) & $4(1-13)$ \\
MCA T2 ASPECT score, median (range) & \\
$\quad$ Left & $6(4-11)$ \\
$\quad$ Right & $5(2-9)$ \\
MCA DWI ASPECT score, median (range) & \\
$\quad$ Left & $5.5(4-10)$ \\
$\quad$ Right & $4.5(2-8)$ \\
Insular involvement, $n$ (\%) & \\
$\quad$ Left & $8(100 \%)$ \\
$\quad$ Right & $5(62.5 \%)$ \\
\hline ASPECT Alberta stroke program early CT score, MRI magnetic resonance \\
imaging, MCA middle cerebral artery, DWI diffusion-weighted imaging, T2 \\
T2-weighted MRI
\end{tabular}

With regard to the effect of stroke laterality on hemodynamic indices under autonomic control, we found no significant differences in heart rate, systolic, diastolic, or mean arterial blood pressure during the period of study between cases of left and right MCA stroke (Table 4), nor in the days preceding (data not shown).

\section{DISCUSSION}

In this study, we show that unilateral MCA stroke in the term newborn has significant effects on autonomic cardiovascular control, as measured by HRV. Specifically, stroke in the right vs. left MCA territory is followed by significantly increased sympathetic tone, independent of other variables. Conversely, left MCA stroke is associated with increased parasympathetic tone, of marginal significance. However, the laterality of the MCA stroke had no significant impact on the systemic hemodynamic variables under autonomic control. Increasing infarct size, regardless of stroke lateralization, is associated with decreasing sympathetic and increasing parasympathetic tone. Surprisingly, in our population injury to either insular region has no significant effect on the autonomic function. Finally, phenobarbital blood levels have significant autonomic effects, increasing sympathetic tone, and decreasing parasympathetic tone. 
Table 4. Comparison of systemic hemodynamic metrics between leftand right-sided stroke groups on the day of HRV analysis

\begin{tabular}{llll}
\hline Metric & $\begin{array}{l}\text { Left MCA stroke, mean } \\
\text { (standard deviation) }\end{array}$ & $\begin{array}{l}\text { Right MCA stroke, } \\
\text { mean (standard } \\
\text { deviation) }\end{array}$ & $P$ value \\
\hline Heart rate & $137.5(12.95) \mathrm{bpm}$ & $130.13(9.95) \mathrm{bpm}$ & 0.11 \\
Systolic BP & $71.63(8.72) \mathrm{mmHg}$ & $71.63(8.72) \mathrm{mmHg}$ & 0.13 \\
Diastolic BP & $47.25(6.27) \mathrm{mmHg}$ & $45.25(6.27) \mathrm{mmHg}$ & 0.24 \\
Mean BP & $57.38(6.95) \mathrm{mmHg}$ & $53.38(6.86) \mathrm{mmHg}$ & 0.13 \\
\hline $\begin{array}{llll}\text { MCA middle cerebral artery, BP blood pressure, bpm beats per minute, } \\
\text { mmHg millimeters mercury }\end{array}$ & & \\
\hline
\end{tabular}

The phenomenon of autonomic dysfunction following vasoocclusive stroke in adults has been known for some time. ${ }^{15-17}$ In general, adult strokes have been associated with an increase in circulating catecholamines, although it remains unclear whether this is the result of increased sympathetic activity, decreased parasympathetic activity, or a change in the balance between the systems. $^{18-20}$ We and others have reported on autonomic dysfunction in newborns with global cerebral hypoxic-ischemic injury. ${ }^{10,21}$ However, to our knowledge, this is the first report of autonomic changes following vaso-occlusive stroke in the newborn. We studied autonomic changes in this model of unilateral MCA stroke for several reasons. First, studies in adult subjects suggest that the majority (but not all) of putative higher cortical autonomic centers are within the middle and anterior cerebral artery territories. ${ }^{2,5}$ The large majority of strokes in the newborn are in the MCA territory. ${ }^{1}$ The neonatal model of stroke differs significantly from the adult in both cerebral and vascular substrate, including the maturational state of cerebral connectivity, as well as the prevalence of often widespread cerebral arteriosclerotic disease in older humans. Unlike our earlier studies in infants with global ischemic brain insults, vaso-occlusive stroke in the newborn is confined to the perfusion territory of a specific artery. We confined our study population to newborns with MCA infarcts, and presumably normal brain tissue elsewhere, in order to allow an internal comparison between the left- and right-sided MCA stroke.

Early understanding considered the major central autonomic centers to be confined to the brainstem and diencephalon, and in a reflexive relationship with the peripheral autonomic system. However, it is now known that a network of cortical and subcortical centers coordinate autonomic function in a system collectively called the central autonomic network. Evidence for both "top-down" and "bottom up" pathways ${ }^{22}$ between higher cortical/subcortical and brainstem autonomic centers has been reported. In newborns with hypoxic-ischemic encephalopathy, a strong association between electrocortical activity and HRV provided further evidence of cerebral cortical modulation of autonomic function. ${ }^{23}$

Animal studies, as well as lesional and cortical stimulation studies in adult humans, suggest that cortical modulation of cardiovascular function may be topographically determined, with sympathetic modulation primarily from the right hemisphere, and parasympathetic modulation from the left hemisphere. ${ }^{24,25}$ Our previous findings in newborns after global hypoxic-ischemic insults and regional brain injury corroborated these findings in adults. $^{10,21}$ Amobarbital injections into the left carotid artery causes tachycardia (suggesting parasympathetic inhibition), while right-sided infusions cause bradycardia. ${ }^{26}$ From another perspective, cerebral injury or dysfunction has been associated with potentially lethal cardiac complications, presumably mediated by the ANS. Sudden unexpected death in epilepsy (SUDEP) $)^{27,28}$ is now understood to result from excessive parasympathetic output, with bradycardia and death, most commonly from left-sided seizures. ${ }^{29}$ Conversely, right-sided stroke has been associated with a stress-related cardiomyopathy (neurogenic stunned myocardium or Takotsubo syndrome) thought to result from chronic sympathetic overactivation, with tachyarrhythmias, and potential death. $^{17}$

Certain findings in our study were unexpected. First, our finding of increased sympathetic tone after irreversible right MCA injury was counterintuitive, given the existing data for sympathetic centers in the right hemisphere. ${ }^{24,25}$ However, this same paradox has been reported in some but not all previous stroke studies. ${ }^{30,31}$ In a rodent model, right hemispheric stroke resulted in a chronic increase in sympathetic activity with resulting chronic systolic dysfunction. ${ }^{30}$ In other rodent models, injury to the right insula caused an increase in heart rate and blood pressure, in some but not all strains. ${ }^{32,33}$ In adult strokes, a pattern of increased sympathetic tone and decreased parasympathetic tone (with tachyarrhythmias) most consistently followed right insular strokes. ${ }^{31}$ The basis for these disparate findings remains unclear.

Another unexpected finding in our study was the lack of an independent association between insular injury on either side and subsequent autonomic dysfunction. Previously both lesional and stimulation studies have implicated the right insula in sympathetic influence, and the left insula in baroreflex function. ${ }^{34}$ The insula is directly connected, likely through both excitatory and inhibitory pathways, to deeper centers in the thalamus, hypothalamus, brainstem, and cerebellum. ${ }^{34,35}$ What mechanisms might underlie the differences in autonomic dysfunction in studies of insular stroke, including the current study? The autonomic changes may result from direct insular cortex injury ${ }^{36,37}$ or dysfunction in downstream insulofugal pathways (excitatory and inhibitory) to deeper brainstem and diencephalon centers, or direct concurrent injury to these brainstem and diencephalon centers. ${ }^{30}$ In a recent study, we described greater HRV attenuation following thalamic and basal ganglia injury compared to injury in the cortical gray and hemispheric white matter. ${ }^{10}$ If descending inhibitory pathways are preferentially injured, lower cardiovascular centers could theoretically become disinhibited and show increased sympathetic activity.

Concurrent direct injury to the major sympathetic output centers in the brainstem and diencephalon is an unlikely explanation for the lack of autonomic effects after insular injury in our study since these centers are outside the MCA supply area. Animal studies have shown that lesions within different sub-regions of the insula have different autonomic effects. ${ }^{38}$ Unfortunately, our sample size precluded a more detailed examination of regional insular injury. The lack of a significant autonomic effect after insular injury is unlikely to be maturational in origin, since the insula is the earliest cortical structure to develop. ${ }^{39}$

We found a significant effect of phenobarbital on autonomic tone in our population. As in an earlier report from a small cohort of epileptic children, ${ }^{40}$ phenobarbital in our cases was associated with enhanced sympathetic tone and a decrease in parasympathetic tone. The mechanism(s) for these autonomic effects of phenobarbital is not clear.

Previous studies have reported on differences in the systemic expression of stroke-mediated autonomic dysfunction. ${ }^{30}$ Both transient and sustained changes in parameters such as blood pressure and heart rate have been described. ${ }^{30}$ In our study, there were no differences in measures of blood pressure and heart rate during the period of our autonomic measurements at around 7 days of age, nor in the days preceding. This disconnect between changes in cardiac autonomic function as measured by HRV and the systemic cardiovascular expression of such changes in this population is intriguing and warrants further study.

This study has several strengths, including the detailed characterization of MRI findings by a neuroradiologist, unilateral injury allowing internal comparison with the opposite intact 
hemisphere, and the use of advanced signal processing of $24 \mathrm{~h}$ of EKG. In addition, we performed our analyses on data at around 7 days of age and at least $48 \mathrm{~h}$ after the last clinical seizure, in order to ensure that regions of injury were committed and that paroxysmal seizures did not contaminate the data. However, our study also had a number of notable limitations. Our relatively small sample size limited the number of variables we could analyze, including other medications. We studied a single $24 \mathrm{~h}$ window of HRV at around 7 days of age, and are unable to comment on the longer-term effects of these strokes. Finally, strokes in regions other than the MCA are far less common and therefore we are unable to comment on the autonomic topography over other cortical regions.

\section{CONCLUSION}

With this report, we extend our earlier lesion-based studies to explore the higher cerebral topography of autonomic modulation in the newborn infant. We examine this question in cases with clearly delineated unilateral injury, within an otherwise normal brain. Based on earlier studies by our group and others, several of the findings in the current study were counterintuitive; this is particularly true of the augmented sympathetic tone following right MCA stroke, as well as the apparent lack of autonomic change after insular injury on either side. Resolving these questions will require further study with a larger sample of equally well-characterized cases.

\section{ACKNOWLEDGEMENTS}

We would like to thank Ms. Sophie Wohlers for her editorial assistance. This study was supported by an internal special purpose fund in the Fetal Medicine Institute at Children's National.

\section{ADDITIONAL INFORMATION}

Competing interests: The authors declare no competing interests.

Publisher's note: Springer Nature remains neutral with regard to jurisdictional claims in published maps and institutional affiliations.

\section{REFERENCES}

1. Inder, T. E. \& Volpe, J. J. Chapter 21-Stroke in the Newborn. Volpe's Neurology of the Newborn 6th edn, 564-589.e567 (Elsevier, Amsterdam, 2018).

2. Benarroch, E. E. in Primer on the Autonomic Nervous System 3rd edn (eds Biaggioni, I., Burnstock, G., Low, P. A. \& Paton, J. F. R.) 9-12 (Academic Press, San Diego, 2012).

3. Thayer, J. F. \& Lane, R. D. Claude Bernard and the heart-brain connection: further elaboration of a model of neurovisceral integration. Neurosci. Biobehav. Rev. 33, 81-88 (2009)

4. Nelson, K. B., Sartwelle, T. P. \& Rouse, D. J. Electronic fetal monitoring, cerebral palsy, and caesarean section: assumptions versus evidence. BMJ 355, i6405 (2016).

5. Kimmerly, D. A review of human neuroimaging investigations involved with central autonomic regulation of baroreflex-mediated cardiovascular control. Auton Neurosci. 207, 10-21 (2017).

6. Beslow, L. et al. Modified Pediatric ASPECTS correlates with infarct volume in childhood arterial ischemic stroke. Front. Neurol. 3, 122 (2012).

7. Govindan, R. N. et al. Detrended fluctuation analysis of non-stationary cardiac beat-to-beat interval of sick infants. 108, 40005 (2014).

8. Govindan, R. B., Massaro, A. N., Niforatos, N. \& du Plessis, A. Mitigating the effect of non-stationarity in spectral analysis-an application to neonate heart rate analysis. Comput. Biol. Med. 43, 2001-2006 (2013).

9. Massaro, A. N. et al. Heart rate variability in encephalopathic newborns during and after therapeutic hypothermia. J. Perinatol. 34, 836-841 (2014).
10. Metzler, M. et al. Pattern of brain injury and depressed heart rate variability in newborns with hypoxic ischemic encephalopathy. Pediatr. Res. 82, 438-443 (2017).

11. Govindan, R. B. et al. A spike correction approach for variability analysis of heart rate in sick infants. Phys. A 444, 35-42 (2016).

12. Nino, G. et al. Premature infants rehospitalized because of an apparent lifethreatening event had distinctive autonomic developmental trajectories. Am. J. Respir. Crit. Care. Med. 194, 379-381 (2016).

13. Hox, J. Applied Multilevel Analysis (TT-Publikaties, Amsterdam, 1995).

14. Muthén LKaM, B. O. Mplus User's Guide (Muthen \& Muthen, Los Angeles, 1998-2017).

15. Constantinescu, V., Matei, D., Costache, V., Cuciureanu, D. \& Arsenescu-Georgescu, C. Linear and nonlinear parameters of heart rate variability in ischemic stroke patients. Neurol. Neurochir. Pol. 52, 194-206 (2017).

16. Simula, S. et al. Effect of middle cerebral artery territory ischemic stroke on QT interval. J. Stroke Cerebrovasc. Dis. 23, 717-723 (2014).

17. Yoshimura, S. et al. Takotsubo cardiomyopathy in acute ischemic stroke. Ann. Neurol. 64, 547-554 (2008)

18. Chen, Z. et al. Brain-heart interaction: cardiac complications after stroke. Circ. Res. 121, 451-468 (2017).

19. De Raedt, S., De Vos, A. \& De Keyser, J. Autonomic dysfunction in acute ischemic stroke: an underexplored therapeutic area? J. Neurol. Sci. 348, 24-34 (2015).

20. Dorrance, A. M. \& Fink, G. Effects of stroke on the autonomic nervous system. Compr. Physiol. 5, 1241-1263 (2015).

21. Schneebaum Sender, N. et al. Cerebral modulation of the autonomic nervous system in term infants. J. Perinatol. 37, 558-562 (2017).

22. Duschek, S., Worsching, J. \& Reyes Del Paso, G. A. Autonomic cardiovascular regulation and cortical tone. Clin. Physiol. Funct. Imaging 35, 383-392 (2015).

23. Vergales, B. D. et al. Depressed heart rate variability is associated with abnormal EEG, MRI, and death in neonates with hypoxic ischemic encephalopathy. Am. J. Perinatol. 31, 855-862 (2014).

24. Janig, W. \& Habler, H. J. Neurophysiological analysis of target-related sympathetic pathways--from animal to human: similarities and differences. Acta Physiol. Scand. 177, 255-274 (2003).

25. Napadow, V. et al. Brain correlates of autonomic modulation: combining heart rate variability with fMRI. Neuroimage 42, 169-177 (2008).

26. Zamrini, E. Y. et al. Unilateral cerebral inactivation produces differential left/right heart rate responses. Neurology 40, 1408-1411 (1990).

27. Buchhalter, J. \& Cascino, G. D. SUDEP: an important cause of premature mortality in epilepsy across the life spectrum. Neurology 89, 114-115 (2017).

28. Li, J., Ming, Q. \& Lin, W. The insula lobe and sudden unexpected death in epilepsy: a hypothesis. Epileptic Disord. 19, 10-14 (2017).

29. Blumhardt, L. D., Smith, P. E. \& Owen, L. Electrocardiographic accompaniments of temporal lobe epileptic seizures. Lancet 1, 1051-1056 (1986).

30. Bieber, M. et al. Stroke-induced chronic systolic dysfunction driven by sympathetic overactivity. Ann. Neurol. 82, 729-743 (2017).

31. Cheung, R. T., Hachinski, V. C. \& Cechetto, D. F. Cardiovascular response to stress after middle cerebral artery occlusion in rats. Brain Res. 747, 181-188 (1997).

32. Butcher, K. S. \& Cechetto, D. F. Insular lesion evokes autonomic effects of stroke in normotensive and hypertensive rats. Stroke 26, 459-465 (1995).

33. Hachinski, V. C., Oppenheimer, S. M., Wilson, J. X., Guiraudon, C. \& Cechetto, D. F. Asymmetry of sympathetic consequences of experimental stroke. Arch. Neurol. 49, 697-702 (1992)

34. Marins, F. R. et al. Functional topography of cardiovascular regulation along the rostrocaudal axis of the rat posterior insular cortex. Clin. Exp. Pharmacol. Physiol. 43, 484-493 (2016).

35. Chen, C. H. et al. Impact of supratentorial cerebral hemorrhage on the complexity of heart rate variability in acute stroke. Sci. Rep. 8, 11473 (2018).

36. Cheshire, W. P. Jr. \& Saper, C. B. The insular cortex and cardiac response to stroke. Neurology 66, 1296-1297 (2006).

37. Walter, U. et al. Insular stroke is associated with acute sympathetic hyperactivation and immunodepression. Eur. J. Neurol. 20, 153-159 (2013).

38. Oppenheimer, S. M., Gelb, A., Girvin, J. P. \& Hachinski, V. C. Cardiovascular effects of human insular cortex stimulation. Neurology 42, 1727-1732 (1992).

39. Alcauter, S., Lin, W., Keith Smith, J., Gilmore, J. H. \& Gao, W. Consistent anterior-posterior segregation of the insula during the first 2 years of life. Cereb. Cortex 25, 1176-1187 (2015).

40. Hallioglu, O., Okuyaz, C., Mert, E. \& Makharoblidze, K. Effects of antiepileptic drug therapy on heart rate variability in children with epilepsy. Epilepsy Res. 79, 49-54 (2008). 